\title{
n-3 poly-unsaturated fatty Acid (n-3PUFA) and neuroprotection: Is docosahexaenoic acid (DHA) involved in glutamate uptake by astrocytes?
}

Barbara Grintal ${ }^{1}$

Gaelle Champeil-Potokar ${ }^{1}$

Philippe Guesnet ${ }^{1}$

Monique Lavialle ${ }^{1}$

Isabelle Denis ${ }^{1}$

Sylvie Breton ${ }^{2}$

\section{NuReLiCe,}

INRA,

Jouy-en-Josas,

France

2. Saint-Hubert,

Rungis,

France

The high amount of DHA (the main n-3PUFA) in brain cell membranes depends on n-3PUFA dietary intake. High $\mathrm{n}$-3PUFA intakes reduce the occurrence of neurodegenerative diseases such as Alzheimer's disease and have a protective effect on experimentally induced excitotoxicity. A high amount of DHA in cell membranes may thus confer to the brain a better resistance to insults. We recently showed that the amount of DHA in membrane phospholipids influences astrocytes physiology. Thus, the neuroprotective effect of n-3PUFA may rely on astrocyte functions involved in neuron protection and synaptic repair. Glutamate scavenging, a major task of astrocytes preventing excitotoxic damage, is under the influence of lipid signalling. We thus hypothesized that a high brain DHA content resulting from adequate n-3PUFA intakes may promote the glutamate uptake by astrocytes.

We have explored the influence of membrane n-3PUFA status on glutamate uptake by astrocytes, on in vivo and in vitro models. In vivo, we compared the amount of astrocytic glutamate transporters GLAST and GLT-1 (western blot), in the cortex and hippocampus from n-3 supplemented or $\mathrm{n}-3$ deficient rats, from birth to 4 months old. In vitro, we compared GLAST and GLT-1 expression (western blot), $3 \mathrm{H}$-aspartatve uptake and the capture of a bolus of glutamate, on astrocytes cultured in DHA-supplemented or control medium. In vivo and in vitro, n-3 PUFA supplementation induced a strong increase in the amount of DHA in membrane phospholipids (measured in phosphatidylethanolamine and phosphatidylcholine, by Gas Chromatography) as compared to n-3 deprived cells or rats. However, the DHA increase had no major consequences on glutamate transporters or uptake, in basal conditions. We are currently exploring the potential influence of DHA in conditions requiring enhanced glutamate c-apture and rapid addressing of the transporters to the membrane. 